The University of Maine

\title{
DigitalCommons@UMaine
}

Marine Sciences Faculty Scholarship

School of Marine Sciences

$12-15-1993$

\section{A Modification of the Response Method of Tidal Analysis}

\author{
R. A. Hyde \\ Neal Pettigrew \\ University of Maine - Main, nealp@maine.edu
}

Follow this and additional works at: https://digitalcommons.library.umaine.edu/sms_facpub

\section{Repository Citation}

Hyde, R. A. and Pettigrew, Neal, "A Modification of the Response Method of Tidal Analysis" (1993). Marine Sciences Faculty Scholarship. 7.

https://digitalcommons.library.umaine.edu/sms_facpub/7 


\title{
Self-Consistency Between Wind Stress, Wave Spectrum, and Wind-Induced Wave Growth for Fully Rough Air-Sea Interface
}

\author{
Gérard Caudal
}

\author{
Centre de Recherches en Physique de l'Environnement Terrestre et Planétaire, Centre Universitaire, Vélizy, France
}

\begin{abstract}
When the flow at the air-sea interface is aerodynamically rough (i.e., for wind speeds above $7.5 \mathrm{~m}$ $s^{-1}$, typically), then form drag on the roughness elements accounts for virtually all the stress. Assuming such a situation, we seek a self-consistent solution for the wind stress in the sense that the wind stress entering the models of sea spectrum and wind-induced growth rate is constrained to be equal to the wind stress obtained through integration of form drag over the wavenumber space. Using the models by Donelan and Pierson (1987) for both fully developed sea spectrum and short-scale wind-induced wave growth rate, we find self-consistent, firmly constrained solutions with roughness lengths in good agreement with Charnock's formulation (namely, $z_{0}$ proportional to $u^{* 2}$ ), with a constant of proportionality which is of a magnitude very close to experimental values reported in the literature. Thus it appears that there is consistency between our knowledge of the surface stress, wind input, and spectral shape. In contrast, when the short-scale wind-induced growth rate is replaced by the one proposed by Plant (1982), the roughness length $z_{0}$ is found to become extremely sensitive to small fluctuations of either wind-induced growth rate or sea-wave spectral level. A poorly constrained and highly fluctuating rather than deterministic value of Charnock's coefficient would then be expected at full development. Numerous confirmations of Charnock's relationship performed in the field at or near full development tend to support Donelan and Pierson's approach for wind-induced growth rate at short scales. The model was also tested in versions in which the viscous drag was tentatively parameterized, and the conclusions reported here were qualitatively unaffected.
\end{abstract}

\section{INTRODUCTION}

The exchange of momentum at the air-sea interface and its relation to both the wind speed and the spectral properties of the sea surface have been the subject of active research. Experiments at sea have progressively improved our description of that coupling [Smith, 1980; Large and Pond, 1981; Geernaert and Katsaros, 1986]. In a review on the subject, Donelan [1990] describes the sea surface as behaving like an approximately smooth surface for wind speeds below $2.5 \mathrm{~m} \mathrm{~s}^{-1}$, while fully rough flow is usually considered in the open ocean for wind speeds above $7.5 \mathrm{~m} \mathrm{~s}^{-1}[\mathrm{Wu}$, 1980; Donelan, 1990]. In smooth flow the roughness elements are buried within the viscous sublayer, and the roughness depends only on the imposed stress and fluid viscosity. With increasing friction velocity $u^{*}$, the viscous sublayer thins until the roughness elements begin to interact directly with the turbulent outer flow (transitional regime). Finally, for $u^{*}$ increasing further, the flow becomes fully aerodynamically rough. In that situation, form drag on the roughness elements accounts for virtually all the stress [Phillips, 1977, p. 193; Donelan, 1990]. Form drag exerted by the atmosphere on a spectral component of the sea surface is a function of both the growth rate imparted by the wind to the spectral component and the amplitude of that spectral component.

If the statement that form drag accounts for virtually all the stress is correct, then the wind stress determined by summing the form drag exerted by the atmosphere on every spectral component should approximately match the wind stress determined experimentally in the surface layer. Such an integration was performed by several authors [Janssen, 1989, 1991; Nordeng, 1991], who were thus able to show

Copyright 1993 by the American Geophysical Union.

Paper number 93JC02351. 0148-0227/93/93JC-02351\$05.00 how the wave age influences the drag coefficient of the air-sea interface. With those approaches, however, the momentum flux to the short gravity-capillary waves needed to be parameterized separately because of the restricted range of validity of the expression for wave growth rate, which was based on Miles's [1957] mechanism. To this end, Janssen [1989, 1991] parameterized the effect of short gravitycapillary waves through Charnock's [1955] formulation, whereas Nordeng [1991] used Kitaigorodskii's [1973] approach. In those studies therefore, the integration of form drag over the sea spectrum formally concerned only gravity waves long enough for the approximations of Miles's theory to hold. The question then arises as to whether some of the empirical expressions currently proposed in the literature for the wave growth rate could have a broader spectral validity in such a way that the momentum flux to short gravitycapillary waves could also be adequately modeled. In that case (and provided that we would also have a realistic model of the sea spectrum), integration of momentum flux could be performed over the whole spectrum (from swell up to capillary waves), thus yielding the wind stress without the need for an additional parameterization of the stress exerted upon gravity-capillary waves.

The aim of this paper is to propose such an approach. It is intended to deal with fully aerodynamically rough situations only, so that the molecular viscous stress at the surface can be ignored, and for that reason only situations with winds larger than a minimum (which we take as $7 \mathrm{~m} \mathrm{~s}^{-1}$ ) will be considered. For a given wind speed at a reference level (say, 10-m altitude), the models describing the growth rate (and to a lesser extent the sea spectrum) are usually dependent upon the friction velocity and thus depend upon the wind stress. We shall therefore seek a self-consistent solution in the sense that the wind stress used to determine the windinduced growth rate and equilibrium sea spectrum is con- 
strained to be the same as the wind stress obtained by integrating the form drag.

In section 2 we describe the methodology and the model employed. In section 3 we give the results of the computation obtained for fully developed seas with various wind conditions. In the light of those results, we then compare the abilities of two different types of parameterizations of the wave growth rate to give realistic self-consistent solutions.

\section{THE MOdeL}

Throughout this paper we shall limit ourselves to considering fully developed seas. Neutral stability is assumed, and as a consequence, the mean wind velocity at height $z$ in the surface boundary layer (above the viscous sublayer) is approximated by the logarithmic profile

$$
U_{(z)}=\frac{u^{*}}{\kappa} \ln \left(\frac{z}{z_{0}}\right)
$$

where $u^{*}$ is friction velocity, $z_{0}$ is roughness length, and $\kappa$ $(=0.41)$ is von Karman's constant.

The wind is supposed to be blowing with steady strength and direction, given as $\mathrm{U}_{10}$ at a height $z=10 \mathrm{~m}$, and no current is present. Then determining $u^{*}$ is equivalent to determining any of the following quantities:

$$
\begin{aligned}
& \text { Roughness length } \quad z_{0}=(10 \mathrm{~m}) \exp \left(-\frac{\kappa \mathrm{U}_{10}}{u^{*}}\right) \\
& \text { Drag coefficient at } 10 \mathrm{~m} \quad \mathrm{C}_{10}=\frac{u^{* 2}}{\mathrm{U}_{10}^{2}}
\end{aligned}
$$$$
\text { Wind stress } \quad \tau=\rho_{a} u^{* 2}
$$

where $\rho_{a}$ is air mass density $\left(=1.3 \mathrm{~kg} \mathrm{~m}^{-3}\right)$.

In this section we show how we compute the wind stress $\tau$ (or, equivalently, any of the other quantities $u^{*}, z_{0}$, and $C_{10}$ ) as a function of the $10-\mathrm{m}$ wind speed $U_{10}$ by integrating the stress over the whole spectrum (from capillary waves to swell).

\subsection{Method for Determining the Wind Stress $\tau$}

The total wind stress $\tau$ at the sea surface is usually expressed as the sum of three terms [e.g., Janssen, 1991; Nordeng, 1991]:

$$
\tau=\tau_{w}+\tau_{t}+\tau_{\text {visc }}
$$

In that expression, the two first terms of the right-hand side are the wave-induced stress $\tau_{w}$ and the turbulent stress $\tau_{t}$, whereas the third term $\tau_{\text {visc }}$ is the stress communicated by the air to the water as a tangential viscous stress. When the air flow over a wavy surface is fully aerodynamically rough, then form drag on the roughness elements accounts for virtually all the stress [Phillips, 1977; Donelan, 1990]. The form drag as defined by Phillips [1977] is the drag exerted by the individual roughness elements, in which molecular viscosity is unimportant. In the present study we shall assume such a fully rough situation, which implies that viscous drag is regarded as negligible. It is usually admitted that the flow in the surface layer in the open ocean is aerodynamically fully rough for $U_{10} \geq 7.5 \mathrm{~m} \mathrm{~s}^{-1}$ [Wu, 1980; Donelan, 1990]. For this reason we shall limit our investigation to situations with wind speed larger than a minimum, which we take here as $7 \mathrm{~m} \mathrm{~s}^{-1}$. The third term $\tau_{\text {visc }}$ will then be neglected.

Nordeng [1991] points out that $\tau_{w}$ must decrease with distance away from the surface, while $\tau_{t}$ must decrease when approaching the surface (see his equation (22) and discussion therewith). Similarly, Janssen [1989] introduces a height-dependent wave-induced stress (see his equation (16)) which is equal to the stress communicated by the air to the wave spectral components with wavenumber $k<k_{\max }$, where $k_{\max }$ is a function of $z$. Thus the distinction between $\tau_{w}$ and $\tau_{t}$ is related to the altitude at which they are measured or, conversely, to the size of the smallest roughness elements considered. In that sense, for a given altitude, $\tau_{w}$ is the stress communicated to the long waves, whereas the turbulent stress $\tau_{t}$ must be ultimately communicated to the short waves (although in an unpredictable manner). In practice, the separation between the short-scale and the large-scale domains is taken at the wavenumber at which Miles's [1957] theory ceases to be valid. The condition of validity of Miles's theory is that the critical level (i.e., the level at which the average wind speed matches the sea wave phase velocity $c$ ) be outside the viscous sublayer, which corresponds to the requirement that $c>5 u^{*}$ [Janssen, 1989].

In a previous study Nordeng [1991] computes the wave drag over the interval of wavenumbers such that $c>5 u^{*}$, which permits him to compute $\tau_{w}$. The turbulent stress $\tau_{\mathrm{t}}$ is parameterized separately and added. Note that $\tau_{w}$ usually contributes to a moderate part of the wind stress (20\% of the total stress on the average, according to Nordeng). The major part of wave drag is carried by the short waves (through $\tau_{t}$ ). In contrast to Nordeng's work, we shall compute here the wave drag by performing the integration over the whole spectral domain (from swell to capillary waves). We shall test the assumption that virtually all the wind stress is thereby accounted for. Therefore no additional parameterization of the turbulent stress will be added. At short scale (where Miles's theory is not applicable), we shall rely on expressions of the wave growth rate which have been proposed in the literature (based on experiments and numerical calculations).

Under those circumstances, the wind stress $\tau$ can then be obtained by projecting onto the wind direction and then integrating the momentum flux applied to every spectral component of the sea waves [e.g., Plant, 1982]:

$$
\tau \approx \tau_{w}=\rho_{w} \int_{0}^{\infty} \int_{0}^{2 \pi} \beta_{w} \omega F(k, \varphi) \cos \varphi k d k d \varphi
$$

where $\rho_{w}$ is water mass density; $(k, \varphi)$ are polar coordinates in the wavenumber space, with $\varphi=0$ corresponding to the wind direction (which is also the direction of wind stress since the wind was supposed to blow in a steady direction); $F(k, \varphi)$ is the two-dimensional sea spectrum; $\omega$ is angular frequency; and $\left.\beta_{w}=(1 / F)(\partial F / \partial t)\right)$ is the exponential growth rate of the waves in response to the wind. Note that all the wave components (from capillaries to swell) must be included in (3); otherwise (3) would only provide a lower bound to $\tau$. From $\tau$, the other quantities $u^{*}, z_{0}$, and $C_{10}$ may be deduced from $(2 a),(2 b)$, and $(2 c)$.

Several processes occurring subsequently in the water will modify the wave spectrum (nonlinear wave-wave interactions, dissipation by viscosity, wave breaking). For the 
shorter wave components, microscale breaking may even remove the surplus wave energy almost immediately after it has been communicated to the wave. These processes, however, only lead to a redistribution of momentum within the sea (ultimately leading to the generation of currents) but do not participate in the momentum transfer from air to water (except indirectly by reshaping the sea surface).

\subsection{Model for the Sea Spectrum}

Donelan and Pierson [1987] (hereinafter referred to as DP) have proposed a two-dimensional model for fully developed seas that covers the whole spectral domain from swell to capillary waves. At low wavenumber (i.e., for $k$ smaller than 10 times the spectral peak $k_{p}$ ), it is based upon a previous model which was proposed by Donelan et al. [1985] (hereinafter referred to as DHH) for fetch-limited situations, of which DP took the full development limit (i.e., $\mathrm{U}_{10} / C_{p}=$ 0.83 , where $C_{D}$ is the phase speed at spectral peak). DP's low-wavenumber spectrum is expressed as follows:

$$
\begin{aligned}
& F(k, \varphi) \\
& =\frac{1.62 \times 10^{-3} \mathrm{U}_{10}}{k^{3.5} g^{0.5}} \exp \left(-\left(\frac{k_{p}}{k}\right)^{2}\right) 1.7^{\Gamma} h \operatorname{sech}^{2}(h \varphi)
\end{aligned}
$$

where

$$
\begin{gathered}
k_{p}=g /\left(1.2 \mathrm{U}_{10}\right)^{2} \\
\Gamma=\exp \left[-1.22\left(\left(\frac{k}{k_{p}}\right)^{0.5}-1\right)^{2}\right] \\
h=1.24 \quad 0<k / k_{p}<0.31 \\
h=2.61\left(k / k_{p}\right)^{0.65} \quad 0.31<k / k_{p}<0.90 \\
h=2.28\left(k_{p} / k\right)^{0.65} \quad 0.90<k / k_{p}<10
\end{gathered}
$$

and where $g\left(=9.81 \mathrm{~m} \mathrm{~s}^{-2}\right)$ is the acceleration of gravity.

Note that DP modified slightly the spreading parameter $h$ of the spectral model compared to that in the initial version of DHH (see the domain of (7c) compared to DHH's equation (9.2)). This corresponds to a directional spread broader than the one of DHH between $k=2.56 k_{p}$ and $k=$ $10 k_{p}$, and this is in accordance with the more recent study by Banner [1990].

For the high-wavenumber portion $\left(k>10 k_{p}\right)$, DP express equilibrium between wind input and dissipation, which allows them to obtain the following expression for the sea spectrum along the wind direction $(\varphi=0)$ :

$$
\begin{gathered}
F(k, \varphi=0)=k^{-4}\left[\frac{0.194}{\alpha(k)} \frac{\rho_{a}}{\rho_{w}}\left(\frac{U(\pi / k)}{C(k)}-1\right)^{2}\right. \\
\left.-\frac{4 \nu_{w} k}{\alpha(k) C(k)}\right]^{1 / n(k)} U(\pi / k)>C(k),[\quad]>0 \\
F(k, \varphi=0)=0 \quad \text { otherwise }
\end{gathered}
$$

In this expression, $C(k)$ is the sea wave phase speed, and $\nu_{w}$ is the kinematic viscosity of water (which we shall take here as $0.013 \mathrm{~cm}^{2} \mathrm{~s}^{-1}$, which corresponds to a water temperature of $10^{\circ} \mathrm{C}$ ). DP computed $U(\pi / k)$ (i.e., the wind at height $\pi / k$ ) from the $10-\mathrm{m}$ wind by using an eqmpirical expression for the drag coefficient that was yalid for a fully developed sea. The quantities $\alpha(k)$ and $n(k)$ are functions of $k, g$, and the surface tension/density ratio for water (taken as $74 \mathrm{~cm}^{3} \mathrm{~s}^{-2}$ ). Those functions depend upon five adjustable coefficients. DP tuned those five coefficients so as to get the best fit to the observed radar backscatter at $\mathrm{Ku}$ band while adhering to the constraints of getting a spectrum close to a $k^{-4}$ power law near $10 k_{p}$ and matching the low-wavenumber spectral level at $10 k_{p}$.

From (8), DP extrapolate the sea spectrum to all directions $\varphi$ in an ad hoc fashion, allowing it to fit the crosswind backscatter measurements (see their equations $(11 c)$, and (12) and discussion therewith).

In this paper we take the same approach as DP with only one difference, which lies in the way $U(\pi / k)$ is determined. In order to determine $U(\pi / k)$ from $\mathrm{U}_{10}$, DP used an empirical expression for the neutral drag coefficient near full development which allowed them to retrieve the wind profile. In contrast, it is the intention of this study to compute the drag coefficient self-consistently (through (3)) instead of relying on an empirical formulation. Assuming a neutral condition, once the $10-\mathrm{m}$ wind is known, one can determine the vertical wind profile under the condition that either $\mathrm{C}_{10}$, $\tau, u^{*}$, or $z_{0}$ is known.

Assume, for example, that $u^{*}$ is known; then (from (1)), one determines $U(\pi / k)$ through

$$
U(\pi / k)=\mathrm{U}_{10}+\frac{u^{*}}{\kappa} \ln \left(\frac{\pi}{k(10 \mathrm{~m})}\right)
$$

\subsection{Model for the Wind-Induced Growth Rate}

We now have to evaluate the wind-induced growth rate $\beta_{w}$ in (3). DP reviewed various expressions proposed in the literature for $\beta_{w}$. Following the results of the numerical calculation by Al-Zanaidi and Hui [1984], they argued that the appropriate reference wind was the one taken at some height above the roughness elements that is related to their scale. Then, relying on observations by Larson and Wright [1975], they took the following expression for $\beta_{w}$ :

$$
\beta_{w}(k, \varphi=0)=\omega \frac{\rho_{a}}{\rho_{w}} A(\mu-1)^{2}
$$

where $A=0.194$, and $\mu$ is expressed as a function of the wind speed $U(\pi / k)$ at altitude $\pi / k$ and phase speed $C(k)$ through

$$
\mu=U(\pi / k) / C(k)
$$

Expressions (10) and (11) for $\beta_{w}$ were then used by DP to get the aforementioned equilibrium expression (8).

Note that Larson and Wright's measurements were performed by observing the Bragg microwave backscatter and allowed sampling of water wavelengths between 0.7 and 7 $\mathrm{cm}$. Their method thus appears to be well representative of the short scales and was therefore retained by DP. Its validity may, however, be questioned for the other side of the spectral domain. Moreover, for the lowest wavenumbers (say, $k=0.03$, which corresponds to a wavelength $\lambda \approx 200$ $\mathrm{m}), U(\pi / k)$ would be the wind speed at an altitude $(=100 \mathrm{~m}$ in this example) that may well be above the surface layer and would thus be irrelevant. For those wavenumbers it is more appropriate to use observations performed at long wavelengths. Hsiao and Shemdin [1983] (hereinafter referred to 

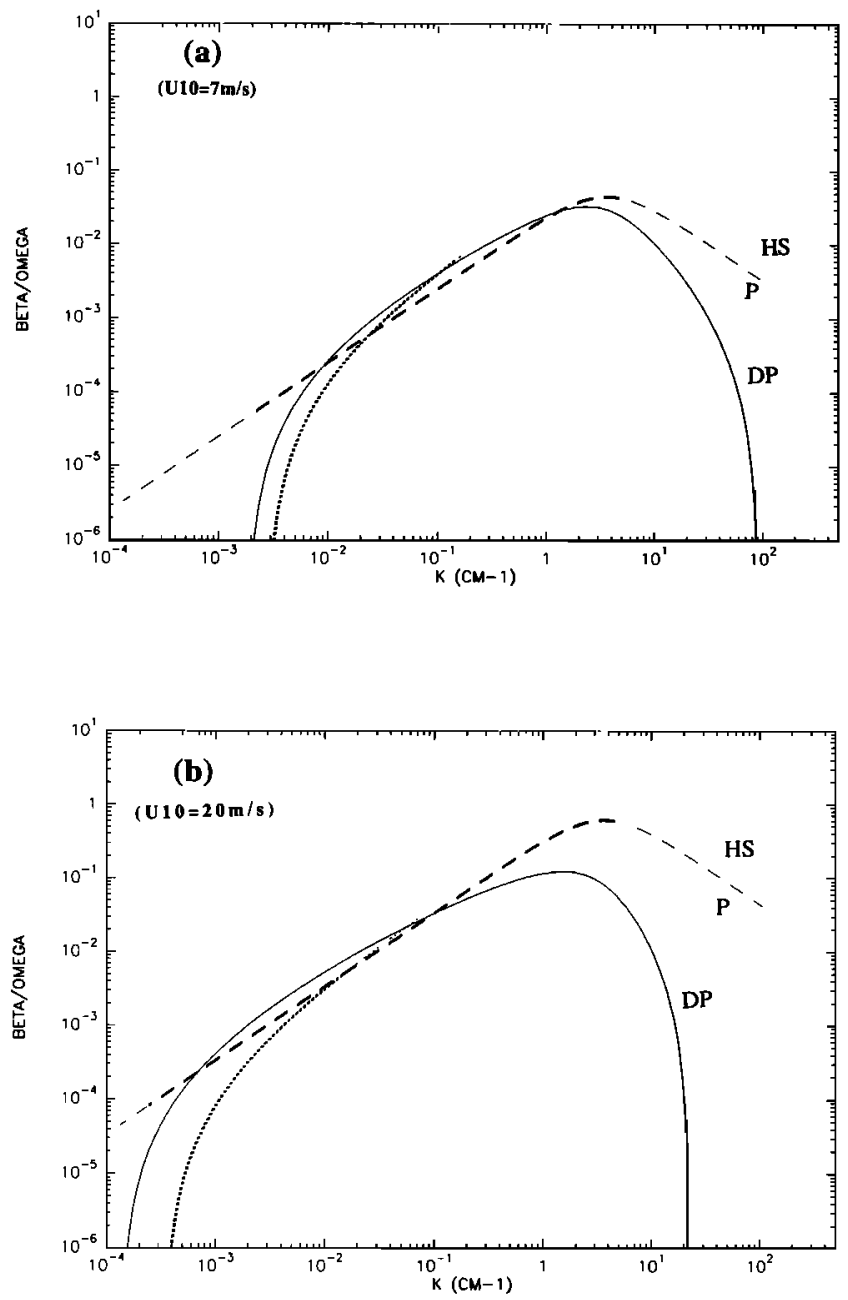

Fig. 1. Ratio between wind-induced growth rate $\beta_{w}$ and sea wave angular frequency $\omega$ as a function of wavenumber in the wind direction. Solid curve is Donelan and Pierson's [1987] model (DP), dotted curve is Hsiao and Shemdin's [1983] model (HS), and dashed curve is Plant's [1982] model (P). The thick parts of the curves representing HS's and Plant's models refer to the range of validity given by the authors. (a) $U_{10}=7 \mathrm{~m} \mathrm{~s}^{-1}$; (b) $U_{10}=20 \mathrm{~m} \mathrm{~s}^{-1}$.

as HS) performed measurements of the wind-induced growth rate at long wavelengths by using a wave follower. Those authors found an expression similar to (10), except that this time they got a good fit to their data by setting $A=0.12$, with

$$
\mu=0.85\left(\mathrm{U}_{10} / C(k)\right)
$$

(where $U_{10}$ is the $10-\mathrm{m}$ wind speed), with a range of validity covering the interval $1<\mu<7.4$.

In the present study we used the wind-induced growth rate given by (10) with DP's version (from Larson and Wright's observations) at high frequencies (11) and HS's version at low frequencies (12). Continuity was achieved by taking the separation between both domains at the wavenumber at which the two versions coincide (this joining frequency is obviously wind dependent).

Figure 1 illustrates the behavior of the wind input growth rate $\beta_{w}$ (normalized for $\omega$ ) for $\mathrm{U}_{10}=7 \mathrm{~m} \mathrm{~s}^{-1}$ (Figure $1 a$ ) and $\mathrm{U}_{10}=20 \mathrm{~m} \mathrm{~s}^{-1}$ (Figure $1 b$ ) as a function of wavenumber as given by DP (our (11)) and by HS (our (12)). Although in our model we compute the roughness length self-consistently, Figure $1 a$ and $1 b$ (given merely for illustrative purpose) have been built assuming Charnock's formulation for the roughness length, $z_{0}=0.014 u^{* 2} / g$ [Charnock, 1955; Garratt, 1977]. Comparing HS's and DP's formulations, one notices for both Figure $1 a$ and Figure $1 b$ that the former gives a smaller $\beta_{w}$ at low wavenumber and a larger $\beta_{w}$ at high wavenumber. Therefore taking HS's formulation at low $k$ and DP's formulation at high $k$ amounts to taking each time the lower of the two formulations. The wavenumber at which both models coincide is between $k=0.05 \mathrm{~cm}^{-1}$ and $k$ $=0.1 \mathrm{~cm}^{-1}$, depending on wind speed. For $U_{10}=20 \mathrm{~m} \mathrm{~s}^{-1}$, it turns out that the transition wavenumber falls outside the range of validity of HS's model (i.e., $1<\mu<7.4$; thick part of the curve in Figure 1). Equation (12) thus needed to be extrapolated somehow for high wind (up to $\mu \approx 12.7$ for $\mathrm{U}_{10}$ $=20 \mathrm{~m} \mathrm{~s}^{-1}$ ). Although such an extrapolation is not justified, it looks at least reasonable in view of the fact that the slopes of the two curves are close to each other at the crossing point and therefore the transition between both domains occurs rather smoothly. For the sake of comparison, we also indicate in Figure 1 the growth rate given by Plant [1982], namely,

$$
\beta_{w}(k, \varphi=0)=\eta u^{* 2} \omega / C(k)^{2}
$$

with $\eta=0.04 \pm 0.02$, of which the mean value $\eta=0.04$ is taken. The range of validity of (13), given as $g /\left(2 \pi \mathrm{U}_{10}\right) \leq$ $(\omega / 2 \pi) \leq 20 \mathrm{~Hz}$ by Plant, also appears as a thick curve in Figure 1.

It can be seen that Plant's and HS's formulations give quite similar behaviors for high wavenumbers $(k>0.02$ $\mathrm{cm}^{-1}$ ), although Plant's version is smaller by a few decibels. In that high-wavenumber portion, DP's version becomes considerably smaller than both other versions, especially at high wind speeds (Figure $1 b$ ).

For waves traveling in directions not aligned with the wind, the same model for $\beta_{w}$ is taken (10) except that $\mu$ is now replaced by $\mu \cos \varphi$. Furthermore, if $(\mu-1)$ happens to be negative, $\beta_{w}$ is set to zero.

\subsection{Description of the Iterative Scheme}

In order to determine the wind stress for a given wind at full development, we need to perform the integration expressed in (3). For this purpose, expressions for both $F(k, \varphi)$ and $\beta_{w}(k, \varphi)$ have been specified above. In section 2.2 , we have shown how we determine $F(k, \varphi)$ from $U_{10}$ for a fully developed sea. Note that the additional knowledge of $u^{*}$ was also required to determine the wind profile from which $U(\pi / k)$ could be computed (9). In section 2.3 , the model for $\beta_{w}(k, \varphi)$ was given, and again both $\mathrm{U}_{10}$ and $u^{*}$ were required (in (11), $U(\pi / k)$ is determined from (9), and therefore $u^{*}$ as well as $\mathrm{U}_{10}$ must be known).

We therefore use the following iterative scheme. For a given wind speed $\left(U_{10}\right)$, we first choose an arbitrary value for $u^{*}$ (practically, we start with $u^{*}$ corresponding to Charnock's formulation $z_{0}=0.0144 u^{* 2} / g$, which combined with (2a) gives an equation for $u^{*}$ which is solved iteratively). We then compute $\tau$ through (3), which yields a new value for $u^{*}$ through $(2 c)$. The process is then repeated with this new $u^{*}$ until convergence is achieved.

\subsection{Assessment of the Assumption that Viscous Drag Is Negligible}

When stating that virtually all the stress is supported by form drag, we assumed that the molecular viscous stress 
directly exerted by the air on the water at the interface contributed to a negligible part of the stress. In order to assess the validity of that assumption, we alternatively followed the description given by Smith [1988], who casts the roughness height of the sea surface $z_{0}$ into two parts:

$$
z_{0}=z_{c}+z_{s}
$$

where $z_{c}$ is the aerodynamic roughness height and is due to the presence of waves, and $z_{s}$ is the roughness length for a smooth surface:

$$
z_{s}=0.11\left(\nu / u^{*}\right)
$$

where $\nu\left(=14 \times 10^{-6} \mathrm{~m}^{2} \mathrm{~s}^{-1}\right)$ is the dynamic viscosity of air.

The additional roughness length $z_{s}$ represents the effect of molecular viscous stress exerted by the air on the water. Unlike Smith, who takes $z_{c}$ as given by the formula of Charnock (i.e., $z_{c}$ proportional to wind stress), we determined $z_{c}$ at each iteration, as seen above (from (3), (2a), and $(2 c))$, and added $z_{s}((14)$ and (15)).

For $U_{10}$ larger than $7 \mathrm{~m} / \mathrm{s}, z_{s}$ appeared to be indeed a small perturbation term, leading to an increase in the selfconsistent roughness length of less than $4.1 \%$ for $U_{10}=7 \mathrm{~m}$ $\mathrm{s}^{-1}$ and less than $0.7 \%$ for $U_{10} \geq 10 \mathrm{~m} \mathrm{~s}^{-1}$. Although the calculation of the roughness length according to (14) and (15) is somehow tentative, it does provide an order of magnitude estimate, allowing us to assess the legitimacy of the assumption that the molecular viscous stress exerted by the air on the water is negligible compared to the total stress. We conclude that for wind speeds above $7 \mathrm{~m} / \mathrm{s}$, the assumption made should provide reasonable accuracy.

\section{Results}

\subsection{Charnock's Coefficient}

The model was run for various values of the wind speed $\mathrm{U}_{10}$, ranging from 7 to $21 \mathrm{~m} \mathrm{~s}^{-1}$. The results may be described in terms of $u^{*}, z_{0}, \mathrm{C}_{10}, \tau$, or a combination of them as a function of $U_{10}$. Here we give the results in terms of Charnock's coefficient, defined as the quantity $m$ entering Charnock's expression:

$$
z_{0}=m u^{* 2} / g
$$

The coefficient $m$ is set arbitrarily to 0.0144 (i.e., the value selected by Garratt [1977]) at the beginning of the run. It is then examined at each iteration until convergence is achieved. Figure 2 shows the evolution of $m$ during the first 15 iterations, otained for the case where $U_{10}=7 \mathrm{~m} \mathrm{~s}^{-1}$. An asymptotic limit of $m \approx 0.0121$ is attained; this is slightly lower than the initial guess.

On the basis of theoretical and empirical concepts, some models for $z_{0}$ as a function of wind and wave conditions have been proposed previously. These were reviewed by Geernaert et al. [1986] and Donelan [1990]. Geernaert et al. compared those modeled values of $z_{0}$ with measured $z_{0}$ for a large variety of wind and wave situations. Among various formulations, they found that Kitaigorodskii's [1973] model performed best in terms of standard deviation between model prediction and observations. Expressed as a function of the direction-averaged wavenumber spectrum $S(k)$, Kitaigorodskii's model may be written

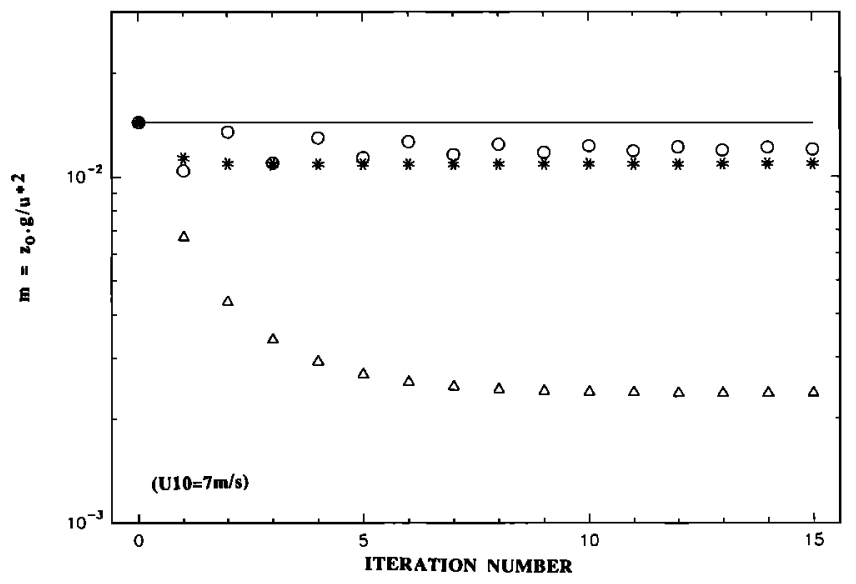

Fig. 2. Evolution of Charnock's coefficient $m=z_{0} g / u^{* 2}$ obtained during the first 15 iterations of our model, where the windinduced growth rate $\beta_{w}$ is given by DP's formulation (open circles) or Plant's formulation (open triangles). The coefficient $m$ obtained when the roughness length $z_{0}$ is given by Kitaigorodskii's approach (17) is also given (asterisks). Solid curve is nominal value $m_{0}=$ 0.0144 .

$$
z_{0}=C_{K}\left[\int_{0}^{\infty} S(k) \exp \left(-2 \kappa c(k) / u^{*}\right) d k\right]^{0.5}
$$

The constant $C_{K}$ was determined empirically by Geernaert et al. as $C_{K} \approx 0.0188$ (in the version in which wind and wave drift currents were not accounted for, which is the case here).

In order to compare our model with Kitaigorodskii's approach, we computed the value of $z_{0}$ corresponding to Kitaigorodskii's formulation (17). The model for sea spectrum $(S(k)$ in (17)) was the same as the one used in our model (see section 2.2). Note that the quantity to be integrated in (17) contains $u^{*}$ and $S(k)$, which (for given $U_{10}$ ) are both dependent upon $z_{0}$. Therefore here again the computation needs an iterative scheme (although convergence occurs after no more than two iterations). With Kitaigorodskii's model, $m$ is found in Figure 2 to converge toward 0.0110 for $\mathrm{U}_{10}=7 \mathrm{~m} \mathrm{~s}^{-1}$.

Finally, we ran the model with the standard assumption (using (3) and the model spectrum from DP), but this time the growth rate $\beta_{w}$ used in (3) is replaced for the highwavenumber domain by the expression proposed by Plant [1982]:

$$
\beta_{w}=\frac{\eta u^{* 2} \omega \cos \varphi}{C(k)^{2}}
$$

with $\eta=0.04 \pm 0.02$, of which we took the mean value, $\eta=$ 0.04. In that case, however, to ensure convergence of our model at high wind speeds, we needed to restrict (18) to $|\varphi|$ $\leq \pi / 2$, setting $\beta_{w}=0$ for $\pi / 2<|\varphi| \leq \pi$. At low wavenumbers, HS's model is still used (equations (10) and (12)), and, as previously, the separation between the low- and highwavenumber domains is taken at the wavenumber at which HS's and Plant's expressions coincide. This time, the resulting Charnock coefficient, also shown in Figure 2, converges toward $m=0.0024$ for $U_{10}=7 \mathrm{~m} \mathrm{~s}^{-1}$, which is a factor of 6 lower than the "nominal" value $\left(m_{0}=0.0144\right)$.

Figure 3 displays the variations of the asymptotic values of 


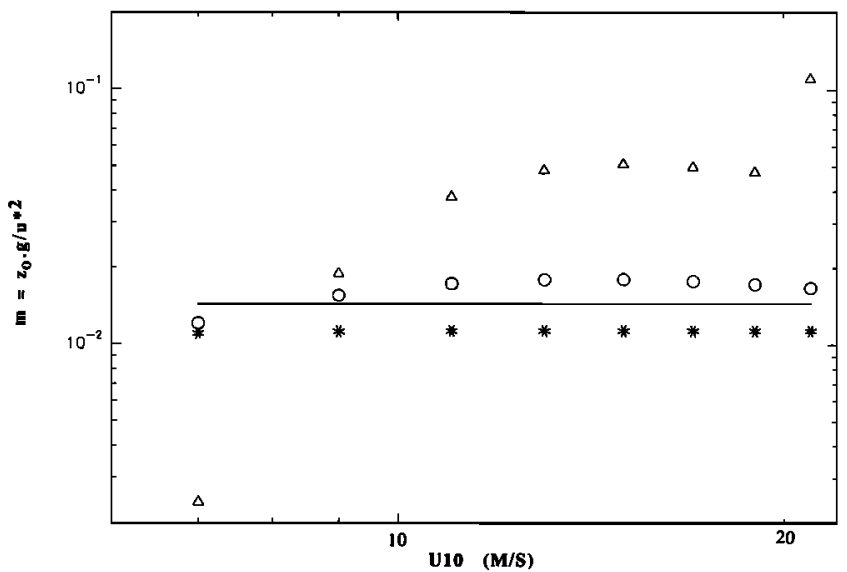

Fig. 3. Asymptotic value of Charnock's coefficient $m=z_{0} g /$ $u^{* 2}$ as a function of wind speed $U_{10}$ obtained from our model with growth rate $\boldsymbol{\beta}_{w}$ given by DP's formulation (open circles) or Plant's formulation (open triangles). The asymptotic value of $m$ obtained through Kitaigorodskii's approach is also given (asterisks). Solid curve is nominal value $m_{0}=\mathbf{0 . 0 1 4 4}$.

the coefficient $m$ as a function of wind speed $U_{10}$ obtained from the three versions described above together with the nominal value $m_{0}=\mathbf{0 . 0 1 4 4}$. We recall that the situation of a fully developed sea is assumed in all cases. It is seen that for the two first versions, $m$ is little affected by the amplitude of wind speed $\mathrm{U}_{10}$ over a wide range of wind speeds ( 7 to $21 \mathrm{~m}$ $\mathbf{s}^{-1}$ ), as might be expected from Charnock's formulation that $z_{0}$ is proportional to $u^{* 2}$. Over the whole range of wind speed, both the model using (3) with $\beta_{w}$ given by DP and Kitaigorodskii's model yield a coefficient $m$ which remains within $\pm 25 \%$ of $m_{0}$. In contrast, the model using (3) with $\beta_{w}$ given by Plant gives a value of $m$ which is from six times lower to more than seven times larger than $m_{0}$.

Numerous observations have led to various estimates for the Charnock constant, with significant scatter $(m=0.0144$ [Garratt, 1977], 0.011 [Smith, 1980], 0.0185 [Wu, 1980], and 0.0192 [Geernaert et al., 1986]. Donelan [1990] retains the value selected by Garratt, $m_{0}=0.0144$, as a reasonable estimate at full development. In our study computations of $m$ either through our standard description (through (3) with $\beta_{w}$ given by DP at short scales), or through Kitaigorodskii's description give values which are within the range of variability of values reported in the literature at full development. In contrast, by using (3) with $\beta_{w}$ given by Plant [1982] at short scale, the value obtained for $\boldsymbol{m}$ indicates an inconsistency between the spectral model, the wind input growth rates, and the field measurements of $z_{0}$.

It must be noted, however, that Plant's formulation allows the coefficient $\eta$ of (18) to have a large variability, since it is expressed as $\eta=0.04 \pm 0.02$. If the variability of $\eta$ is accounted for, the resulting allowable Charnock coefficient $m$ spans a very large interval. Thus for $U_{10}=7 \mathrm{~m} \mathrm{~s}^{-1}$, Plant's formulation with $\boldsymbol{\eta}=\mathbf{0 . 0 6}$ (the upper bound) would lead to $m=0.030$, which is above the nominal value $m_{0}=$ 0.0144 and a factor of 12.5 higher than the solution with $\eta=$ 0.04 displayed in Figure 3. Similarly, for $U_{10}=21 \mathrm{~m} \mathrm{~s}, \eta=$ 0.02 (the lower bound) leads to $m=0.0086$, which is below $m_{0}$. Plant's formulation is thus compatible with our picture, provided that a suitable value for $\eta$ is chosen within the allowable range $(0.04 \pm 0.02)$ for each wind speed. Indeed, with Plant's formulation for $\beta_{w}$, it turns out that a small variation of $\eta$ (or, equivalently, a small variation of the sea wave spectral level) may result in a huge variation in the self-consistent solution for $z_{0}$. To quantify this behavior further, a variation of $\eta$ by only $\pm 1 \%$ in (18) around its nominal value $\left(\eta_{0}=0.04\right)$ gives a variation of $m$ by $\pm 13 \%$ for $\mathrm{U}_{10}=7 \mathrm{~m} \mathrm{~s}^{-1}$. As a comparison, when using $\beta_{w}$ from DP, a variation of the constant $A$ in (10) by $\pm 1 \%$ in the same condition gives a variation of $m$ of no more than $\pm 2 \%$. Over a narrow interval around $U_{10}=20 \mathrm{~m} \mathrm{~s}^{-1}$, Plant's formulation for $\beta_{w}$ even yields two solutions for $m$, which differ from each other by about a factor of 2 . This is the reason for the discontinuity of $m$ which may be seen in Figure 3 around $20 \mathrm{~m} \mathrm{~s}^{-1}$ with Plant's formulation. Such highly singular behavior does not occur with DP's formulation for $\beta_{w}$. The description with $\beta_{w}$ given by DP provides a very robust self-consistent determination of $z_{0}$, whereas Plant's formulation gives a value of $z_{0}$ which is expected to behave in a highly erratic fashion.

In order to explain the large variability obtained with Plant's formulation one might invoke the inconsistency that Plant's formula of the growth rate is different from DP's formula, which is contained in the spectral form at high wavenumber (equation (8)). We have therefore also tested the model in a version in which Plant's formula replaced DP's formula both in the expression of the growth rate and in the spectral form at high wavenumber (equation (8)). As a result, however, the variability of the coefficient $m$ became even larger (at $7 \mathrm{~m} \mathrm{~s}^{-1}, m$ was vanishingly small, and convergence could be achieved only through the inclusion of viscosity according to the method of section 2.5 , while at 21 $\mathrm{m} \mathrm{s}^{-1}, m$ was more than 40 times $m_{0}$ ).

A more fundamental reason for the difference in the behaviors of our model using either Plant's or DP's expressions for $\beta_{w}$ is the following. If, in the course of the iterative process described in section 2.4 , the stress $(\tau)_{n}$ obtained through (3) at iteration $n$ is higher than the previous value $(\tau)_{n-1}=\rho_{a} u_{n-1}^{* 2}$, then the new $u^{*}$ value $u_{n}^{*}=\left(\tau_{n} / \rho_{a}\right)^{0.5}$ has to be increased accordingly. With Plant's formulation, this results in an increased $\beta_{\mathrm{w}}$ (through (18)) which, after integration of (3) at iteration $(n+1)$, will give a new wind stress $(\tau)_{n+1}$, which is further increased in comparison with $(\tau)_{n}$. Ultimately, if the sea spectrum were kept fixed, the iterative scheme could not converge at all, for the simple reason that with both $\beta_{w}$ and $\tau$ being proportional to $u^{* 2}$, (3) could be simplified by dividing both sides by $u^{* 2}$. The iterative process is somewhat stabilized by the sea spectral model, which is slightly dependent upon $u^{*}$ at high wavenumber (through $U(\pi / k)$ in (8)) and also by the different formulation of $\beta_{w}$ and also by the different formulation of $\beta_{w}$ at low wavenumber (equations (10) and (12)). Convergence can thus be reached, although in a slow and poorly deterministic fashion. In contrast, with DP's formulation for $\beta_{w}$, in the same circumstance (i.e., $\left.(\tau)_{n}>(\tau)_{n-1}\right)$, the increase of $u_{n}^{*}$ compared to $u_{n-1}^{*}$ implies a modification of the wind profile which (for a given $U_{10}$ ) leads to a decrease of $U(\pi / k)$ and thus a decrease of $\beta_{w}$ (equations (10) and (11)). The consequence is that the next iteration will give a reduced $\tau$ (i.e., $\tau_{n+1}<\tau_{n}$ ), which provides stabilization of the iterative process. Those different behaviors appear clearly in Figure 2, wher a damped oscillatory behavior occurs for DP's version and monotonous behavior occurs for Plant's version. 
Another reason DP's expression of $\beta_{w}$ provides a more stable behavior in our model is the following. Let us assume for simplicity the classical $k^{-4}$ power law for the spectrum at high wavenumber combined with Plant's expression for the growth rate. Then the main problem in calculating the surface stress from the wave-induced stress is that a logarithmic singularity arises, as pointed out by Janssen [1991] (see his equation (30) and discussion therewith). Thus the wave-induced stress becomes unbounded when all waves are taken into account. DP's model for $\beta_{w}$ does not suffer from this drawback because the growth rate vanishes for sufficiently large frequency. In practice, the logarithmic singularity is still avoided here, even with Plant's expression for $\boldsymbol{\beta}_{w}$, because our spectral model (equation (8)) drops off faster than the $k^{-4}$ law beyond a certain wavenumber, but the fact that $\beta_{w}$ vanishes at high wavenumbers may contribute in part to the more stable behavior of our model when DP's formulation of the growth rate is used.

The consequence of those fundamentally different properties is that with Plant's model for $\beta_{w}$, the self-consistent solution for $z_{0}$ is very poorly determined, as in an illconditioned problem. In that circumstance, one would expect an erratic behavior of $z_{0}$, with huge fluctuations of $z_{0}$ in response to insignificant fluctuations of sea spectrum. In contrast, with DP's formulation one would expect a firmly constrained problem, with a relationship between $z_{0}$ and $u^{* 2}$ fairly well represented by Charnock's formula (as can be seen in Figure 3). Charnock's formulation was corroborated by numerous experiments as cited above. The various estimates of $m$ determined at or near full development tend to organize themselves in a deterministic rather than a chaotic manner. Moreover, those observations tend to converge toward a value of the coefficient $m$ which is consistent with the one obtained here with DP's formulation for $\beta_{w}$ without any ad hoc tuning. Consistency of Kitaigorodskii's approach also tends to support the relevance of the spectral model used in this study. Within the assumptions made in this study, DP's formulation of $\beta_{w}$ (equations (10) and (11)) thus seems to provide a better consistency with roughness length observations at full development than does Plant's formulation (equation (18)).

\subsection{Spectral Distribution of Wave Drag}

In the course of the integration of wind stress over the wave spectrum, it is possible to distribute the stress among the various spectral bands in order to compare the amount of stress supported by waves of different scales. To this end, once convergence was achieved, we divided the wavenumber range into bins, with equal widths in terms of $\log (k)$. The bin size was chosen so as to get three bins per decade in the $k$ space. The histograms giving the stress supported by each spectral bin are displayed in Figure 4 for $U=7 \mathrm{~m} \mathrm{~s}^{-1}$ and in Figure 5 for $U=20 \mathrm{~m} \mathrm{~s}^{-1}$. Our standard model with DP's formulation for $\beta_{w}$ was used. In both Figure 4 and Figure 5, together with the histogram, we show the section through the wavenumber spectrum in the wind direction $(\varphi=0)$. At $\mathrm{U}_{10}=7 \mathrm{~m} / \mathrm{s}$, the maximum wind stress per decade is found to occur for $k \approx 0.1-0.5 \mathrm{~cm}^{-1}$ (i.e., wavelength $\lambda \approx 12-60$ $\mathrm{cm})$. For $\mathrm{U}_{10}=20 \mathrm{~m} \mathrm{~s}^{-1}$ the maximum occurs near $k \approx$ $0.02-0.2 \mathrm{~cm}^{-1}$ (i.e., $\lambda \approx 30 \mathrm{~cm}$ to $3 \mathrm{~m}$ ) and is thus displaced toward larger wavelengths. Note, however, that the vertical scales for wind stress in Figures 4 and 5 differ from each other by a factor of 10 . It is noticeable that in both cases, the wind stress at $k<10 k_{p}$ is insignificant. Finally, beyond $k \approx$ $2 \mathrm{~cm}^{-1}$ (i.e., for $\lambda \leq 3 \mathrm{~cm}$ ), the wind stress also contributes to a negligible portion of the total stress. This means in particular that the constraint of consistency performed in this study is weak as concerns the parameterization of the growth rate of waves with $\lambda \leq 3 \mathrm{~cm}$.

\subsection{Effect of the Orbital Motion of the Long Waves}

Since most of the stress is carried by short waves (with wavelengths of a few tens of centimeters to a few meters), the effect of the orbital motion of the long waves should in principle be taken care of. This has not been done in this study, where only average quantities were considered. In order to quantify the error committed, we have done some tests in a crude two-scale approach by assuming that the long wave is a sine wave with wavenumber equal to the peak wavenumber $k_{p}$ and height variance equal to $H=\iint F(k, \varphi)$ $k d k d \varphi$. For a given patch of the sea surface, we considered the local rest frame and computed the angular frequency $\omega$ of short waves with the acceleration of gravity $g$ replaced by the vector sum of $g$ and the inertial orbital acceleration (neglecting Coriolis acceleration). Also, in that frame, $\mathrm{U}_{10}$ was replaced by an effective wind speed $\mathrm{U}_{10_{\mathrm{eff}}}=\mathrm{U}_{10}-u$, where $u$ is the horizontal orbital velocity at the surface. Using DP's formulation for $\beta_{w}$, we computed the total stress $\tau$ locally according to those modified expressions for $\omega$ and $U_{10}$ and then averaged it over the orbital positions. We treated a case with uniform spectral density of the short waves along the long sine wave and a case in which the short wave spectral density $F(k, \varphi)$ had a sinusoidal modulation, with spectral density being twice as large at the crests as at the troughs (to account for hydrodynamic long wave-short wave interactions). In all cases, the Charnock coefficient $m$ was found to be slightly smaller than in the standard version. The largest difference occurred for the case with nonuniform short-scale spectral density. In that case, compared with DP's standard case of Figure $3, m$ was found to be lower by 12.7 and $5.7 \%$ for wind speeds $U_{10}$ of 7 and $21 \mathrm{~m} \mathrm{~s}^{-1}$, respectively. Those estimates, which ignore the disturbance of the airflow by the long wave, are crude and aim only at quantifying the error committed. They tend to indicate that the effect of the orbital motion of the short waves riding on long waves should not fundamentally alter the results presented above.

\section{Conclusion}

We have used a model describing the fully developed sea spectrum over the full wavenumber space together with a model of the wind input growth rate. For a given wind speed at a reference altitude $(10 \mathrm{~m})$, we have sought a selfconsistent solution for the wind stress in the sense that the wind stress entering the models of sea spectrum and windinduced growth rate was constrained to be equal to the wind stress obtained through integration of wave drag over the wavenumber space (assuming the wind to be high enough that viscous stress may be neglected). Using the models by Donelan and Pierson [1987] for both fully developed sea spectrum and short-scale wind-induced wave growth rate together with Hsiao and Shemdin's [1983] model for largescale wind-induced wave growth rate, we found self- 


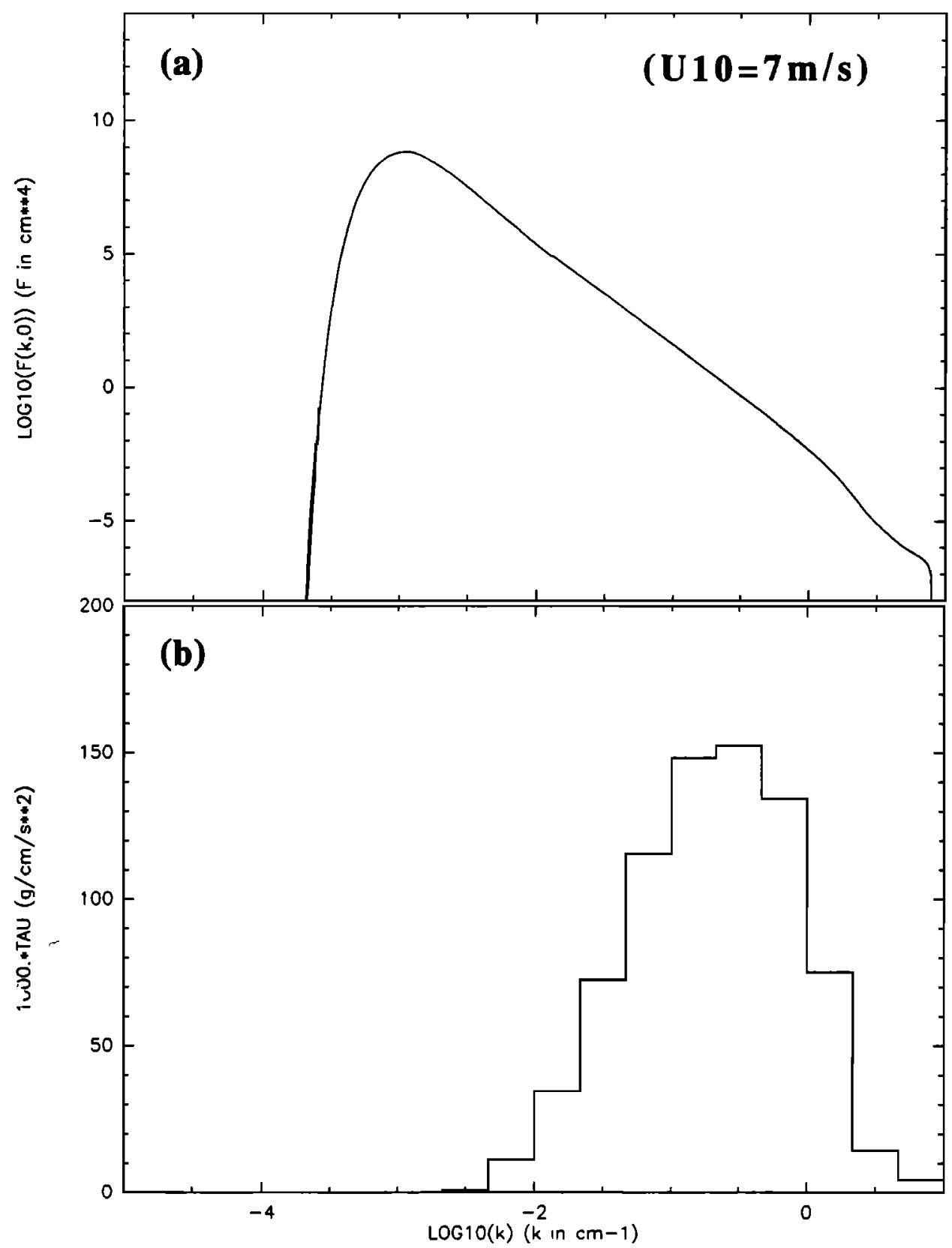

Fig. 4. (a) Section through the wavenumber spectrum in the wind direction ( $\varphi=0$ ) for $U_{10}=7 \mathrm{~m} \mathrm{~s}^{-1}$ (DP's model is used, with $U(\pi / k)$ determined self-consistently from our approach). (b) Corresponding histogram showing the wind stress supported by each spectral bin.

consistent solutions with roughness lengths in good agreement with Charnock's [1955] formulation (namely, $z_{0}$ proportional to $u^{* 2}$ ). Over the wind speed range $7 \mathrm{~m} \mathrm{~s}^{-1} \leq$ $U_{10} \leq 21 \mathrm{~m} \mathrm{~s}^{-1}$, the constant of proportionality between $z_{0}$ and $u^{* 2}$ was of a magnitude comparable to experimental values reported in the literature. This result did not require any ad hoc tuning of the model parameters. Thus the most remarkable conclusion of this paper is that there is now consistency between our knowledge of the surface stress, wind input, and spectral shape.

When the short-scale wind-induced growth rate from DP was replaced by the one proposed by Plant [1982], the roughness length $z_{0}$ was found to become extremely sensitive to small fluctuations of either wind-induced growth rate or sea wave spectral level. A poorly constrained and highly fluctuating rather than deterministic value of Charnock's coefficient would then be expected at full development. Numerous confirmations of Charnock's relationship performed in the field at or near full development seem to support DP's rather than Plant's formulation at short scales.

This work was done with the assumption that form drag accounts for virtually all the stress, and the work is therefore limited to situations of aerodynamically rough flow ( $U_{10} \geq$ $7.5 \mathrm{~m} \mathrm{~s}^{-1}$, typically). Viscous drag was therefore assumed to be of little importance. The model was tested in versions in which the viscous drag was tentatively parameterized, and the conclusions reported here were qualitatively unaffected.

Possible further developments of this work could involve 


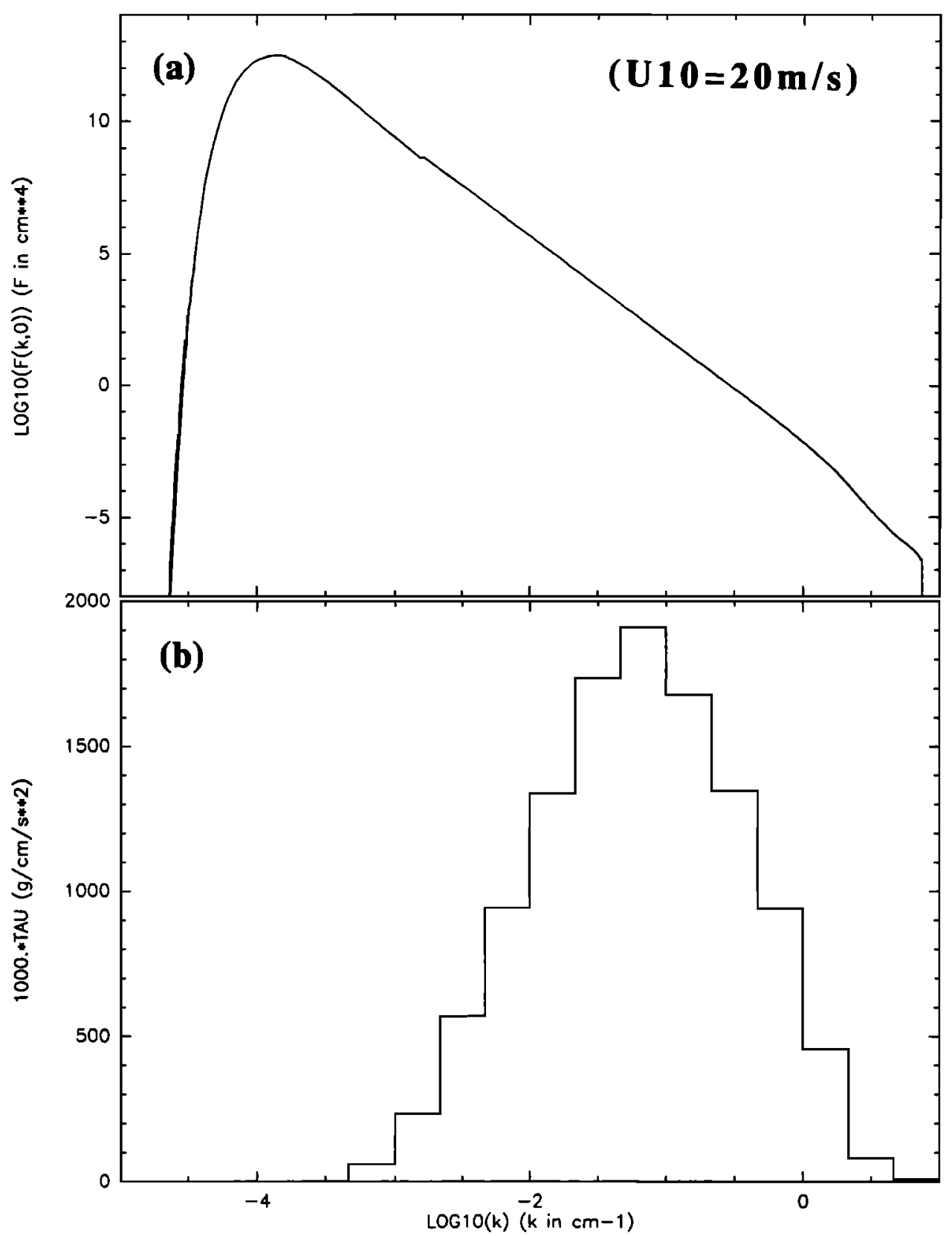

Fig. 5. Same as Figure 4 but for $U_{10}=20 \mathrm{~m} \mathrm{~s}^{-1}$.

the study of fetch-limited situations. A difficulty would then consist in having at one's disposal a realistic quantitative evaluation of the sea spectrum at limited fetch, especially in the short gravity-capillary range, where most of the stress occurs. The requirement of self-consistency would provide a useful constraint relating wind stress, wind speed, and sea spectral level in the short gravity range for a given fetch. Since radar scatterometry of the sea surface is sensitive to the short gravity-capillary waves, such constraints would help us interpret the radar cross-section response to various wind and fetch situations.

Acknowledgments. The author wishes to thank Danièle Hauser, Alain Weill, and Laurence Eymard for a lot of useful discussions as well as judicious comments concerning this paper.

\section{REFERENCES}

Al-Zanaidi, M. A., and W. H. Hui, Turbulent airflow over water waves-A numerical study, J. Fluid Mech., 148, 225-246, 1984. Banner, M. L., Equilibrium spectra of wind waves, J. Phys. Oceanogr., 20, 966-984, 1990.

Charnock, H., Wind stress on a water surface, Q.J. R. Meteorol. Soc., 81, 639-640, 1955.

Donelan, M. A., Air-sea interaction, in The Sea, vol. 9, Ocean Engineering Science, edited by B. Le Méhauté and D. M. Hanes, pp. 239-292, John Wiley, New York, 1990.

Donelan, M. A., and W. J. Pierson, Jr., Radar scattering and equilibrium ranges in wind-generated waves with application to scatterometry, J. Geophys. Res., 92, 4971-5029, 1987.

Donelan, M. A., J. Hamilton, and W. H. Hui, Directional spectra of wind-generated waves, Philos. Trans. R. Soc. London, A, Ser. $315,509-562,1985$.

Garratt, J. R., Review of drag coefficients over oceans and continents, Mon. Weather Rev., 105, 915-929, 1977. 
Geernaert, G. L., K. B. Katsaros, and K. Richter, Variation of the drag coefficient and its dependence on sea state, J. Geophys. Res., 91, 7667-7679, 1986.

Hsiao, S. V., and O. H. Shemdin, Measurements of wind velocity and pressure with a wave follower during MARSEN, J. Geophys. Res., 88, 9841-9849, 1983.

Janssen, P. A. E. M., Wave-induced stress and the drag of air flow over sea waves, J. Phys. Oceanogr., 19, 745-754, 1989.

Janssen, P. A. E. M., Quasi-linear theory of wind-wave generation applied to wave forecasting, $J$. Phys. Oceanogr., 21, 1631-1642, 1991.

Kitaigorodskii, S. A., The Physics of Air-Sea Interaction, translated from Russian by A. Baruch, Israel Program for Scientific Translations, Jerusalem, 1973.

Large, W. G., and S. Pond, Open ocean momentum flux measurements in moderate to strong winds, J. Phys. Oceanogr., 11, 324-336, 1981.

Larson, T. R., and J. W. Wright, Wind-generated gravity-capillary waves: Laboratory measurements of temporal growth rates using microwave backscatter, J. Fluid Mech., 70(3), 417-436, 1975.

Miles, J. W., On the generation of surface waves by shear flows, $J$. Fluid Mech., 3, 185-204, 1957.

Nordeng, T. E., On the wave age-dependent drag coefficient and roughness length at sea, J. Geophys. Res., 96(C4), 7167-7174, 1991.

Phillips, O. M., The Dynamics of the Upper Ocean, 2nd ed., 336 pp., Cambridge University Press, New York, 1977.

Plant, W. J., A relationship between wind stress and wave slope, $J$. Geophys. Res., 87(C3), 1961-1967, 1982.

Smith, S. D., Wind stress and heat flux over the ocean in gale force winds, J. Phys. Oceanogr., 10, 709-726, 1980.

Smith, S. D., Coefficients for sea surface wind stress, heat flux, and wind profiles as a function of wind speed and temperature, $J$. Geophys. Res., 93(C12), 15,467-15,472, 1988.

Wu, J., Wind stress coefficients over sea surface near neutral conditions-A revisit, J. Phys. Oceanogr., 10, 727-740, 1980.

G. Caudal, Centre de Recherches en Physique de l'Environnement Terrestre et Planétaire, Centre Universitaire, 10-12 avenue de l'Europe, 78140 Vélizy, France.

(Received April 9, 1993

revised August 19, 1993;

accepted August 19, 1993.) 